\title{
A Robust Watermarking of Medical Image Based on 3D-DFT and Arnold Scrambling In Compressed Domain
}

\author{
Yanfang $\mathrm{Hu}^{1}$ \\ ${ }^{1}$ College of Information Science and Technology, \\ Hainan University, China, \\ 763941952@qq.com \\ Jingbing $\mathrm{Li}^{2}$ \\ ${ }^{2}$ College of Information Science and Technology, \\ Hainan University, China, \\ Jingbingli2008@hotmail.com
}

\author{
Mengxing Huang ${ }^{3}$ \\ ${ }^{3}$ College of Information Science and Technology, \\ Hainan University, China, \\ huangmx09@163.com
}

\begin{abstract}
Currently, the medical information system is meeting many difficulties when conveying information via the network, such as leaking out the patients' information, falsifying medical records, and destroying the structure of medical images etc. For these questions, the new algorithm with ultra-strong robustness is proposed, its innovation lies in perfectly combining digital watermarking technology, which is the domain technology in information security area, the emerging perceptual Hash technology, the classical threedimensional reconstruction technology, information encryption technology, and "the third party" certification together. Firstly, it is important to preprocess the original watermarking by means of Arnold scrambling; secondly, to obtain the needed information by 3D-DFT, we utilize perceptual Hash function to stratify and reduce the dimensions, as well as compress the medical volume data into $8 \times 8$, then the visual feature vector will be gained after reconstructing the three-dimensional image, which is the result of 3D-IDFT; ultimately, employing the bit operation of Hash function between the encrypted watermarking and visual feature vector, we can realize the embedding process. The experiments indicate that the approach is suitable for medical application because of its robustness.
\end{abstract}

Keywords- Perceptual Hash; 3D reconstruction; Visual feature vector; Arnold scrambling;3D-DFT

\section{INTRODUCTION}

In the network age, the medical industry is suffering some unprecedented challenges like illegal copying for medical images, the stolen private information, and the tempering of medical data, etc. In order to establish a well medical environment, it is significant to find a way to solve the questions effectively and efficiently [1].

Currently, the professionals focus more attentions on observing three-dimensional medical data, in that twodimensional images are limited to the viewing angle and the information what we can obtain is not too much, in light of these reasons, to research the medical volume data, is necessary and unavoidable[2] [3].

Perceptual Hash is one way to extract the abstract information, but the message digest can percept the same content as multimedia information, it satisfies some characteristics such as robustness and abstracts, we can realize the compress of medical images and the extracting for visual feature vector via the two features [4].

This article indicates a new algorithm, via threedimensional DFT and Arnold scrambling technology, protects the medical volume data in the Compressed Domain, it is a zero-watermarking technology with no influence for the original volume data, when we embedding the watermarking, furthermore the one belongs to blind watermarking in that the original volume data is not necessary as to extracting digital watermarking, however, the key features for it include that the low computational complexity, ultra-strong robustness and ability to flexibly control the amount of hiding information.

\section{THE FUNDAMENTAL THEORY}

\section{A. Three- dimension Discrete Fourier Transform (3D- DFT)}

In the paper, the 3D-DFT is used to convert time domain signal into a frequency domain, in that many signals in the space, with strong association, will be concentrated to a certain region, or the distribution of the coefficient matrix with certain regularity in frequency domain signal, so we can reduce the number of quantization bits using these rules, and realize the image compression [5]. For the function $f(x, y, z)$, the 3D-DFT is defined as follows:

$$
\begin{aligned}
F\left(\omega_{1}, \omega_{2}, \omega_{3}\right) & =F[f(x, y, z)] \\
& =\int_{-\infty}^{+\infty} \int_{-\infty}^{+\infty} \int_{-\infty}^{+\infty} f(x, y, z) e^{-j\left(\omega_{1} x+\omega_{2} y+\omega_{3} z\right)} d x d y d z
\end{aligned}
$$


According to the transformation, we can know the 3DIDFT as shown below.

$$
\begin{aligned}
f(x, y, z) & =F^{-1}\left(F\left(\omega_{1}, \omega_{2}, \omega_{3}\right)\right) \\
& =\frac{1}{(2 \pi)^{3}} \int_{-\infty}^{+\infty} \int_{-\infty}^{+\infty} \int_{-\infty}^{+\infty} F\left(\omega_{1}, \omega_{2}, \omega_{3}\right) e^{j\left(\omega_{1} x+\omega_{2} y+\omega_{3} z\right)} d \omega_{1} d \omega_{2} d \omega_{3}
\end{aligned}
$$

Where $f(x, y, z)$ is a three dimensional function in the spatial domain, $F\left(\omega_{1}, \omega_{2}, \omega_{3}\right)$ also means one function but in frequency domain; the medical volume data is regarded as many slices, one slice is a two-dimensional image, the size is $\mathrm{M} \times \mathrm{N}$, and $\mathrm{P}$ means the layer number [6].

\section{B. Arnold scrambling Transform (AT)}

Arnold scrambling transformation is regarded as one kind of the encryption techniques, which can be used as preprocessor for digital image hiding and covering. It is able to turn a meaningful watermarking image into a worthless image with owning the certain robustness, and also can improve the performance, as well as the scrambling algorithm overcomes the unrecoverable of random scrambling[7][8].

Two-dimensional Arnold scrambling transformation is defined as follows:

$$
\left[\begin{array}{l}
x^{\prime} \\
y^{\prime}
\end{array}\right]=\left[\begin{array}{ll}
1 & 1 \\
1 & 2
\end{array}\right]\left[\begin{array}{l}
x \\
y
\end{array}\right] \bmod N \quad \quad x, y \in\{0,1,2, \ldots, N-1\}
$$

Where $\mathrm{x}, \mathrm{y}$ is the pixel coordinates of the original space; $\mathrm{x}^{\prime}, \mathrm{y}^{\prime}$ is the pixel coordinates after iterative computation scrambling. $\mathrm{N}$ is the size of the rectangular image, also referred as a step number.

In the paper, we take $64 \times 64$ binary watermarking image, the scrambling number $\mathrm{n}$ is 24 , and the scrambling period $\mathrm{T}$ is equal to 48. Experimental results are showed in Fig .1, Fig .1 (a) is the original watermarking; Fig .1 (b) shows the scrambled watermarking image.

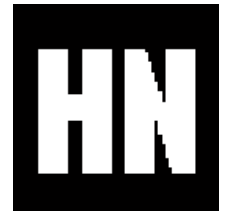

(a)

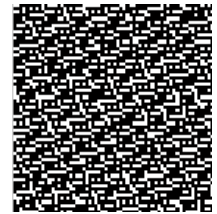

(b)
Figure1. The scrambled watermarking image using Arnold scrambling: (a) the original watermarking; (b) the scrambled watermarking.

\section{Perceptual Hash}

Perceptual Hash is a method to gain the abstract information through a class of one way mappings, which from multimedia presentations to a perceptual Hash value in terms of their perceptual content, and the digital summary is called a perceptual Hash value. It is sensitive to the operation that changing images' perceptual content, in addition, it has great robustness for the manipulation of no reversing the image content, the function of perceptual Hash is as follows.

$$
h=P H()
$$

Where $\mathrm{h}$ indicates a perceptual Hash value, namely the abstract of perceptual content; $\mathrm{PH}$ is the function of perceptual Hash.

\section{THE ALGORITHM}

The original watermarking image is described as: $W=\{w(i, j) \mid w(i, j)=0$ or $1 ; 1 \leq i \leq M 1,1 \leq j \leq M 2\}$. At the same time, we select a medical volume data as the original image. It is described as:

$$
F=\{f(i, j, k) \mid f(i, j, k) \in R ; 1 \leq i \leq M, 1 \leq j \leq N, 1 \leq k \leq P\} . \text { To }
$$
facilitate the operation, we assume $\mathrm{M}=\mathrm{N}, \mathrm{P}$ is the number of slices.

\section{A. The algorithm of visible feature vector extraction}

In the paper, the perception Hash algorithm is used to extract visual feature vector of medical image in order to embed the watermarking. We choose one of 2D slice images and process it based on perceptual Hash algorithm, the specific process is as follows.

Step 1: Reduce the image size

It is the way to maintain the structure, but clear up the high frequency and detail, the size of medical image will be reduced to $8 \times 8$, and the total pixels is 64 .

Step 2: Simplify the colors

The grayscale of shrunken image should be switched from 255 to 64 , translate, there is only have 64 colors among all the pixels

Step 3: Calculate the gray average of 64 pixels.

Step 4: Compared the pixel gray value

We compare each value of pixel gray and the average, let " 1 " represent greater or equal to the average, and " 0 " indicate less than the average.

Step 5: Calculate the Hash value

We combine the results in the step 3 then obtain a 64-bit integer, which is the fingerprint of this image, or the Hash value, namely the visible feature vector, which is what we wanted [9].

The following Table 1 shows the extracted feature vectors of medical volume data using perceptual Hash algorithm, and these medical volume data are subject to different attack, in addition, the table also reflects the relative value of Peak Signal to Noise Ratio (PSNR) and Normalized Crosscorrelation (NC), which is close to 1.0.

In order to verify whether the sequence can be regarded as the characteristic values of the volume data, one effective way is pointed out that comparing the Normalized Crosscorrelation with many different volume data, we deal with those in same way, and extract the corresponding feature vector, which show in Table2. Obviously, the maximum value of correlation coefficient is up to 1.00 which comparing with themselves, the different ones but they looking very much like will generate a relatively great $\mathrm{NC}$ value, and the minimum in close connection with the extremely dissimilar ones. What we can see from the results that the feature vectors can reflect the characteristics accurately, the following images respectively represent 
different volume data named "V1、V2、V3、V4、V5、

V6" , which show in the Fig.2.

TABLE I. THE FEATURE VECTORS OF MEDICAL IMAGE WITH DIFFERENT ATTACKS

\begin{tabular}{|c|c|c|c|c|c|c|c|c|c|c|c|}
\hline Image processing & SNR & $\mathrm{F}(3,5,1)$ & $\mathrm{F}(3,6,1)$ & $\mathrm{F}(4,2,2)$ & $\mathrm{F}(4,2,3)$ & $\mathrm{F}(5,1,3)$ & $\mathrm{F}(5,2,3)$ & $\mathrm{F}(6,1,4)$ & $\begin{array}{c}\text { Pixels } \\
\text { mean }\end{array}$ & $\begin{array}{c}\text { Sequence of } \\
\text { signs }\end{array}$ & $\mathrm{NC}$ \\
\hline Original image & & $5+0.7 \mathrm{i}$ & $8+0.3 i$ & $4+0.5 \mathrm{i}$ & $4+0.1 \mathrm{i}$ & $2.2+0.9$ & $1.7+0.4 \mathrm{i}$ & $2.3+0.7 \mathrm{i}$ & $3.7+0.4 \mathrm{i}$ & 1111000 & 1.0 \\
\hline Gaussian noise & .04 & $5.8+0.7 \mathrm{i}$ & $7.3+0.2 \mathrm{i}$ & $.1+0.3 \mathrm{i}$ & $5.1+0.2 \mathrm{i}$ & $4.3+0.5 \mathrm{i}$ & $4.2+0.1 \mathrm{i}$ & $4.4+0.2 \mathrm{i}$ & $5.8+0.1 \mathrm{i}$ & 1111000 & 1.0 \\
\hline JPEG compression (4\%) & 17.82 & $5.8+0.2 \mathrm{i}$ & $5.7+0.3 \mathrm{i}$ & $4.4+0.7 \mathrm{i}$ & $5.7+0.9 \mathrm{i}$ & $2.4+0.2 \mathrm{i}$ & $2.0+0.8 \mathrm{i}$ & $2.6+0.1 \mathrm{i}$ & $4.2+0.8 \mathrm{i}$ & 1111000 & 1.0 \\
\hline Median filter [3x3] & 22.46 & $5.3+0.5 \mathrm{i}$ & $5.2+0.6 \mathrm{i}$ & $4.7+0.1 \mathrm{i}$ & $5.0+0.9 \mathrm{i}$ & $2.2+0.6 \mathrm{i}$ & $1.7+0.3 \mathrm{i}$ & $2.4+0.6 \mathrm{i}$ & $3.5 .+0.6 \mathrm{i}$ & 1111000 & 1.0 \\
\hline Rotation $\left(8^{\circ}\right)$ & 4.68 & $5.2+0.4 \mathrm{i}$ & $5.6+0.1 \mathrm{i}$ & $.3+0.4 \mathrm{i}$ & $5.4+0.2 \mathrm{i}$ & $1.2+0.4 \mathrm{i}$ & $1.6+0.3 \mathrm{i}$ & $2.6+0.2 \mathrm{i}$ & $3.7+0.1 \mathrm{i}$ & 1111000 & 1.0 \\
\hline Transla & .65 & $5.8+0.8 \mathrm{i}$ & $5.9+0.4 \mathrm{i}$ & $3.9+0.3 \mathrm{i}$ & $5.1+0.4 \mathrm{i}$ & $2.2+0.4 \mathrm{i}$ & $1.6+0.9 \mathrm{i}$ & $2.3+0.4 \mathrm{i}$ & $2.7+0.6 \mathrm{i}$ & 1111000 & 1.0 \\
\hline Distortion factor (20) & 976 & $5.8+0.2 \mathrm{i}$ & $5.2+0.7 \mathrm{i}$ & $3.8+0.5 \mathrm{i}$ & $4.3+0.7 \mathrm{i}$ & $2.0+0.9 \mathrm{i}$ & $2.1+0.5 \mathrm{i}$ & $2.5+0.9 \mathrm{i}$ & $3.7+0.6 \mathrm{i}$ & 1111000 & 1.0 \\
\hline Cropping (3\% from y) & & $5.8+0.6 \mathrm{i}$ & $5.6+0.8 \mathrm{i}$ & $3.9+0.8 \mathrm{i}$ & $5.2+0.5 \mathrm{i}$ & $2.2+0.6 \mathrm{i}$ & $1.6+0.9 \mathrm{i}$ & $2.2+0.3 \mathrm{i}$ & $3.7+0.5 \mathrm{i}$ & 1111000 & 1.0 \\
\hline Cropping (3\% from $\mathrm{x}$ ) & & $5.8+0.1 \mathrm{i}$ & $5.4+0.5 \mathrm{i}$ & $4.7+0.3 \mathrm{i}$ & $4.0+0.3 \mathrm{i}$ & $2.3+0.9 \mathrm{i}$ & $1.0+0.6 \mathrm{i}$ & $2.2+0.5 \mathrm{i}$ & $3.7+0.9 \mathrm{i}$ & 1111000 & 1.0 \\
\hline Cropping (3\% fron & & $5.8+0.3 \mathrm{i}$ & $5.2+0.6 \mathrm{i}$ & $3.9+0.8 \mathrm{i}$ & $5.3+0.7 \mathrm{i}$ & $2.0+0.2 \mathrm{i}$ & $1.5+0.7 \mathrm{i}$ & $2.1+0.3 \mathrm{i}$ & $3.4+0.2 \mathrm{i}$ & 1111000 & 1.0 \\
\hline MRI Scaling (x2) & & $8.8+0.5 \mathrm{i}$ & $8.2+0.7 \mathrm{i}$ & $8.3+0.3 i$ & $8.6+0.7 \mathrm{i}$ & $4.0+0.9 \mathrm{i}$ & $2.6+0.3 \mathrm{i}$ & $4.5+0.6 \mathrm{i}$ & $8.0+0.5 \mathrm{i}$ & 1111000 & 1.0 \\
\hline MRI Scaling (x0.5) & & $1.8+0.2 \mathrm{i}$ & $1.4+0.3 \mathrm{i}$ & $1.2+0.5 \mathrm{i}$ & $1.3+0.7 \mathrm{i}$ & $1.1+0.4 \mathrm{i}$ & $0.8+0.2 \mathrm{i}$ & $0.9+0.1 \mathrm{i}$ & $0.9+0.3 \mathrm{i}$ & 1111000 & 1.0 \\
\hline
\end{tabular}

The unit of transform coefficients is $1.0 \mathrm{e}+005 *$

TABLE II. THE CORRELATION COEFFICIENT WITH DIFFERENT VOLUME DATA (VECTOR LENGTH 64 BIT)

\begin{tabular}{|c|c|c|c|c|c|c|c|}
\hline & V1 & V2 & V3 & V4 & V5 & V6 & V7 \\
\hline V1 & 1.000 & 0.335 & 0.302 & 0.125 & 0.318 & 0.279 & 0.043 \\
\hline V2 & 0.345 & 1.000 & 0.786 & 0.098 & 0.174 & 0.113 & 0.074 \\
\hline V3 & 0.302 & 0.786 & 1.000 & 0.105 & 0.145 & 0.103 & 0.085 \\
\hline V4 & 0.125 & 0.098 & 0.105 & 1.000 & 0.178 & 0.845 & 0.092 \\
\hline V5 & 0.318 & 0.174 & 0.145 & 0.178 & 1.000 & 0.846 & 0.089 \\
\hline V6 & 0.279 & 0.113 & 0.103 & 0.845 & 0.846 & 1.000 & 0.102 \\
\hline V7 & 0.043 & 0.074 & 0.085 & 0.092 & 0.089 & 0.102 & 1.000 \\
\hline
\end{tabular}

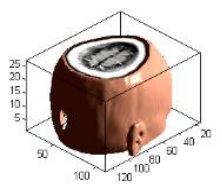

V1

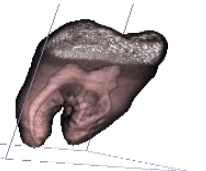

V4

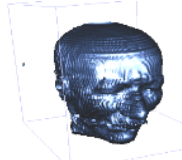

V2

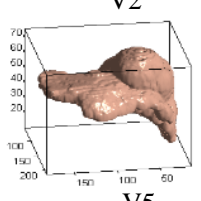

V5

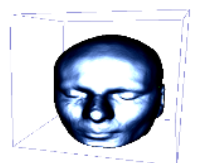

V3

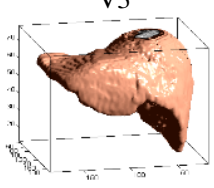

V6
Figure 2. The different medical volume data

\section{B. The embedding algorithm}

In this experiment, we take $64 \times 64$ binary image $\mathrm{W}$, which as watermark image, and the medical volume data, which is $128 \times 128 \times 27$, as carried image. The method to embed watermarking is showed as the following:
- Step1: Acquire the encrypted watermarking image To scramble the watermarking image using Arnold scrambling, we can generate the encrypted watermarking image $B W(i, j)$ as follows:

$$
B W(i, j)=A T(W(i . j))
$$

- Step2: Acquire the feature vector

At first, we adopted 3D-DFT transform to obtain the carried image information [10], then used perceptual Hashing algorithm to extract the information summary so that realized the image compression. At last, we need to realize the threedimensional reconstruction using 3D-IDFT, and the feature vector $V(j)$ is the value of perceptual Hashing h, specific process as following.

$$
F D(i, j, k)=\operatorname{DFT} 3(F(i, j, k))
$$

$$
\begin{gathered}
F I D(i, j, k)=\operatorname{IDFT3}(F D(i, j, k)) \\
h=\operatorname{PH}(\operatorname{FID}(i, j, k)) \\
V(j)=h
\end{gathered}
$$

- $\quad$ Step3: Generate two-valued logic sequence Utilizing and the feature vector $V(j)$ the encrypted watermarking $B W(i, j)$, we can generate the sequence $\operatorname{Key}(i, j)$ :

$$
\operatorname{Key}(i, j)=V(j) \oplus B W(i, j)
$$

In order to extract the important information $\operatorname{Key}(i, j)$ should be stored well. Furthermore, the $\operatorname{Key}(i, j)$ can also be registered to the third part to preserve the ownership of the original image.

\section{The algorithm to extract watermarking}

- Step1: Acquire the feature vector.

This process of acquiring the feature vector $V^{\prime}(j)$ is similar to the above step2 algorithm for embedding of the watermarking [11]. $F^{\prime}(i, j, k)$ is 
the tested image, its feature vector is $V^{\prime}=\left\{v^{\prime}(j) \mid v^{\prime}(j)=0,1 ; \quad 1 \leq j \leq L\right\}$.

$$
\begin{gathered}
F D^{\prime}(i, j, k)=D F T 3\left(F^{\prime}(i, j, k)\right) \\
F I D^{\prime}(i, j, k)=\operatorname{IDFT3}\left(F D^{\prime}(i, j, k)\right) \\
h^{\prime}=P H\left(F I D^{\prime}(i, j, k)\right) \\
V^{\prime}(j)=h^{\prime}
\end{gathered}
$$

- $\quad$ Step2: Extract the watermarking image

$$
B W^{\prime}(i, j)=k e y(i, j) \oplus V^{\prime}(j)
$$

Where, $B W^{\prime}(i, j)$ is the extracted watermarking, $V^{\prime}(j)$ owns to tested image, and $\operatorname{Key}(i, j)$ is obtained from the above process of watermarking embedding.

- Step3: Extract decrypt original watermarking Using the Arnold scrambling inverse transform to restore the extracted watermarking $B W^{\prime}(i, j)$ and get the tested image watermarking $W^{\prime}(i, j)$.

$$
W^{\prime}(i, j)=\operatorname{IAT}\left(B W^{\prime}(i, j)\right)
$$

\section{The algorithm of watermarking detection}

The Normalized Cross-correlation (NC) is used for measuring the quantitative similarity between the extracted and embedded watermarking, which is defined as:

$$
N C=\frac{\sum_{i} \sum_{j} W(i, j) W^{\prime}(i, j)}{\sum_{i} \sum_{j} W^{2}(i, j)}
$$

Where $W(i, j)$ denotes the embedded original watermarking and $W^{\prime}(i, j)$ denotes the extracted original watermarking. The higher the $\mathrm{NC}$ value, the more similarity there is between the embedded and extracted original watermarking.

The Peak Signal to Noise Ratio (PSNR) is used for measuring the distortion of the watermarked image, which is defined as:

$$
P S N R=10 \lg \left[\frac{M N \max _{i, j}(\mathrm{I}(i, j))^{2}}{\sum_{i} \sum_{j}\left(\mathrm{I}(i, j)-\mathrm{I}^{\prime}(i, j)\right)^{2}}\right]
$$

Where $\mathrm{I}(i, j), \mathrm{I}^{\prime}(i, j)$ denote the pixel gray values of the coordinates $(i, j)$ in the original image and the watermarked image, respectively; $\mathrm{M}, \mathrm{N}$ represent the image row and column numbers of pixels, respectively.

\section{EXPERIMENTS}

To verify the effectiveness, we carried out the simulation in Matlab2010a platform [12], and choose some key images. Fig .3(a) shows the 3D medical image; Fig .3(b) indicates the tenth slice of 3D medical image $F=\{f(\mathrm{i}, \mathrm{j}) ; 1 \leq \mathrm{i} \leq 128,1 \leq j \leq 128\} \quad$ Fig $.3(\mathrm{c})$ demonstrates the original binary watermarking $W=\{w(i, j)=0$ or $1 ; 1 \leq i \leq 64,1 \leq j \leq 64\}=\{\mathrm{w}(\mathrm{i}, \mathrm{j})=0$ or $1 ; 1$ $\leqslant \mathrm{i} \leqslant 64,1 \leqslant \mathrm{j} \leqslant 64\}$, these image are not subjected to any attack.

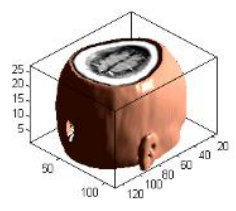

(a)

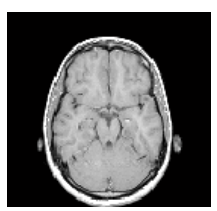

(b)

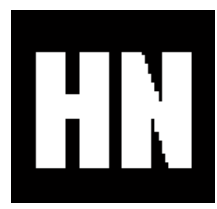

(c)
Figure 3.The medical image without attacking: (a) the original 3D medical image; (b) one of 2D medical image slices; (c) the original binary watermarking image.

The followings are several different types of attacks, including common attacks and geometrical attacks, in order to test the robustness of the new algorithm.

\section{A. Common attacks}

\section{1) Gaussian noise}

The watermarking image is processed via Gaussian noise. Table 3 shows the values of PSNR and NC for medical image with suffering the different degrees of Gaussian noise attack. Fig .4 shows the Gaussian noise strength with $10 \%, \mathrm{PSNR}=3.32 \mathrm{~dB}$, and $\mathrm{NC}=1.00$, the $3 \mathrm{D}$ medical image under the attack is shown in Fig .4(a); As shown in Fig .4(b), the tenth 2D slice of medical volumes data with the same attack; Fig .4(c) shows the extracted watermarking image with $\mathrm{NC}=1.00$. The results prove that our proposed algorithm has strong robustness against Gaussian noise attacks.

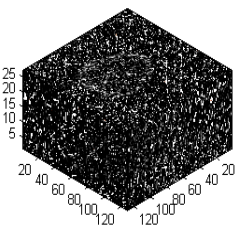

(a)

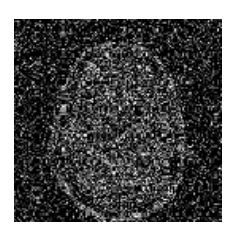

(b)

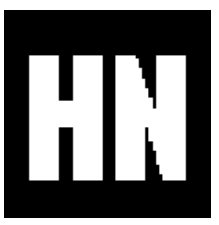

(c)
Figure 4. Under Gaussian noise attacks (10\%): (a) a 3D medical image under noise attack; (b) the tenth 2D medical image slice (c) the extracted watermarking image.

TABLE III. THE PSNR AND NC UNDER DIFFERENT GAUSSIAN NOISE

\begin{tabular}{|c|c|c|c|c|c|c|c|c|}
\hline Gaussian noise $(\%)$ & 1 & 3 & 5 & 10 & 15 & 20 & 25 & 30 \\
\hline PSNR(dB) & 12.51 & 8.05 & 6.03 & 3.32 & 1.78 & 0.84 & 0.09 & -0.04 \\
\hline NC & 1.00 & 1.00 & 1.00 & 1.00 & 1.00 & 1.00 & 1.00 & 1.00 \\
\hline
\end{tabular}

\section{B. Geometrical attacks}

\section{1) Scaling attacks}

Table4 demonstrates the medical image is subjected to rotation attack with different index, the value of PSNR and $\mathrm{NC}$ is shown in the following table. We select a rotation attack with clockwise 40 in the Fig .5 to research. Fig .5(a) shows the 3D medical image rotated and clockwise by 40 , the PSNR of the rotated medical image is $11.01 \mathrm{~dB}$. Fig .5(b) indicates the tenth slice of 3D medical image under rotation attack. Fig .5(c) indicates that the extracted watermarking with $\mathrm{NC}=0.79$. The results 
show that the watermarking algorithm also has stronger robustness against rotation attacks than others.

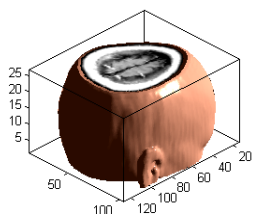

(a)

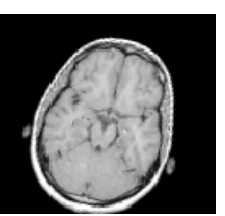

(b)

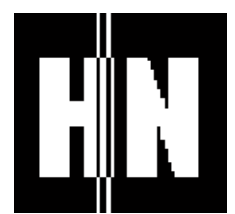

(c)
Figure 5. Under rotation attacks (angle is $40^{\circ}$ ): (a) a 3D medical image under rotation attack; (b) the tenth slice of medical volume data; (c) the extracted watermarking image.

TABLE IV. THE PSNR AND NC UNDER ROTATION ATTACKS WITH DIFFERENT FACTORS.

\begin{tabular}{|c|c|c|c|c|c|c|c|c|}
\hline Rotation attack & $5^{\circ}$ & $10^{\circ}$ & $15^{\circ}$ & $20^{\circ}$ & $25^{\circ}$ & $30^{\circ}$ & $35^{\circ}$ & $40^{\circ}$ \\
\hline PSNR $(\mathrm{dB})$ & 16.54 & 13.96 & 12.98 & 12.44 & 12.04 & 11.68 & 11.33 & 11.01 \\
\hline $\mathrm{NC}$ & 1.00 & 1.00 & 0.92 & 0.85 & 0.85 & 0.85 & 0.83 & 0.79 \\
\hline
\end{tabular}

\section{2) Cropping attacks}

The cropping attacks are added to the watermarking image for validating the effectiveness of our proposed algorithm. The medical image suffered cropping attack with different axis ( $\mathrm{Y}$ and $\mathrm{X})$ in Table5, Fig .6(a) shows that the 3D medical image cropping from $\mathrm{Z}$ axis with the ratio of $12 \%$. Moreover, Fig .6(b) shows the tenth slice of 3D medical image. Fig .6(c) shows that the extracted watermarking image with $\mathrm{NC}=1.00$. The results show that the watermarking algorithm has strong robustness against cropping attacks.

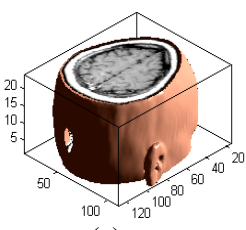

(a)

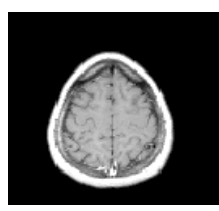

(b)

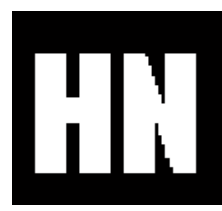

(c)
Figure 6. Under cropping attacks (from the $\mathrm{Z}$ axis, 12\%): (a) a 3D medical image with cropping attack; (b) one of medical image slices(c) the extracted watermarking image.

TABLE V. THE PSNR AND NC UNDER CROPPING ATTACKS FROM Y AND

\begin{tabular}{|c|c|c|c|c|c|c|c|c|c|c|c|}
\hline Cropping attack (\%) & 2 & 4 & 6 & 8 & 10 & 12 & 14 & 16 & 18 & 20 \\
\hline $\mathrm{Z}$ axis & $\mathrm{NC}$ & 1.00 & 1.00 & 1.00 & 1.00 & 1.00 & 1.00 & 1.00 & 1.00 & 1.00 & 1.00 \\
\hline $\mathrm{Y}$ axis & $\mathrm{NC}$ & 0.98 & 0.96 & 0.94 & 0.94 & 0.91 & 0.81 & 0.81 & 0.81 & 0.81 & 0.81 \\
\hline
\end{tabular}

\section{CONCLUSION}

The paper points out an efficient and effective algorithm based on compressed domain, not transform domain, the advantages lie in applying the core technology and the emerging technology, which called digital watermarking and perception Hash, furthermore it owns the unique characteristics of summaries, uniqueness, invisibility, sensitivity, security and robustness comparing with some traditional algorithms. The experiments indicate that the algorithm is able to resist any attacks like conventional attacks and geometric attacks. It is worth researching for medical volume data in the medical area, even in the information security field.

\section{ACKNOWLEDGMENT}

This work is supported by the National Natural Science Foundation of China (No: 61263033) 、 the NSF of Hainan Province of China (No: 60894)、 the Institutions of Higher Learning Scientific Research Special Project of Hainan Province(Hnkyzx2014-2) and the International Science and Technology Cooperation Project of Hainan (KJHZ2014-16).

\section{REFERENCES}

[1] M. Unoki, R. Miyauchi, "Reversible Watermarking for Digital Audio Based on Cochlear Delay Characteristics," In Proceedings of the 2011 Seventh International Conference on Intelligent Information Hiding and Multimedia Signal Processing, Oct. 2011, pp. 314-317.

[2] Wang Xijin;Fan Linxiu, "The Application Research of MD5 Encryption Algorithm in DFT Digital Watermarking." Physics Procedia, Vol.25, 1264-1269,2012.

[3] Marsh, JA;Wohlrab, GF, "Blind extraction and security analysis of spread spectrum hidden watermarks," Conference on Cyber Sensing, CYBER SENSING 2012, Baltimore, MD,2012.

[4] Zheng, Jiang Yong, "A DCT-BASED Digital Watermarking Algorithm for Image." Industrial Control and Electronics Engineering (ICICEE), 2012 International Conference on, Xi'an, China,2012.

[5] Li Tu, Juan $\mathrm{Hu}$, Chi Zhang, Gang Liu, "Image Encryption Algorithm Based on Arnold Transformation and Logistic Mapping”, AISS, vol. 4, no. 23, pp.282-289, 2012.

[6] Xiong, Gang Proquest, "Distributed Synchronization and Spectrum Sensing in Cognitive Radio Networks," UMI Dissertation Publishing;BiblioBazaar, Vol.38, 2012.

[7] Erozan, Ahmet Turan;Baskir, Subutay Giray;Ors, Berna, "Hardware/Software codesign for watermarking in DCT domain." Signal Processing and Communications Applications Conference (SIU), $201321^{\text {st }}, 981367012,2013$.

[8] Liu Tao, "Digital Image Scrambling Improved Algorithm Based on Three DimensionsArnold Transformation," Science Technology and Engineering, 1671-1819,6-1580-04,2010.

[9] Coatrieux, G. Le Guillou, C. Cauvin, J.-M. Roux, "Reversible Watermatking for knowledge Digest Embedding and Reliability Control in Medical Images", IEEE Transactions on Information Technology in Biomedicine, Vol. 2, No. 13, pp.158-165, 2009.

[10] Alar Kuusik, Enar Reilent, Ivor Loobas, Marko Parve, "Software Architecture for Modern Telehealth Care Systems", AISS, Vol. 3, No. 2, pp. $141 \sim 151,2011$.

[11] Xin De Sun. "A Novel Digital Watermarking Algorithm for Medical Color Image". Advanced Materials Research, 2013, 2534(756)

[12] Ajay Goel, Rupesh Gupta. "Improved Digital Watermarking Techniques and Data Embedding In Multimedia”. International Journal on Computer Science and Engineering, 2010, 2(2). 